\title{
Kidney Sensitivity to the Non-Toxic Fraction F1 in Physiological and Non-Physiological State
}

\author{
Type of article: Conference abstract \\ Nadjia Bekkari, Fatima Laraba Djebari \\ USTHB, Faculty of Biological Sciences, Laboratory of Cellular and Molecular Biology, 16111, BP32, \\ El Alia, Bab Ezzouar, Algiers, Algeria \\ Corresponding Author: flaraba@hotmail.com
}

\begin{abstract}
:
Background: Scorpion venom contains several bioactive components that could be useful in therapeutics.

Methods: In this study, the effects of the repeated administration of the nontoxic fraction of Androctonus australis hector (Aah) venom after carcinogenesis induced by N-Nitrosodiethylamine (DEN) were investigated on rats. Metabolic parameters and oxidative stress markers were evaluated in sera and kidney.

Results: The obtained result revealed that DEN had disturbed the metabolic activities (an increase of transaminases and LDH activities) and had led to oxidative stress (a significant increase of NO and MDA levels and depletion of GSH rate) compared to the control. However, repeated injection of F1 shows a kind of modulatory effect on metabolism and oxidative stress. It shows a variable effect on metabolic markers (turn ASAT activity to normal level, recovered ALAT, and activities). A decrease of MDA level in sera and kidney and an increase of GSH level in the kidney were observed.

Conclusion: F1 fraction purified from Aah venom contains many bioactive components. These biomolecules lead to enzymatic activity changes and to modulate oxidative stress in the kidney in normal and carcinogenic model. These changes seem to be beneficial in the pathological case.

Keywords: Aah Venom, Bioactive Components, F1 Fraction, Carcinogenesis, Oxidative Stress.
\end{abstract}

\section{Conflict of interest statement}

This article is a conference abstract presented at the International Congress on Health Sciences and Medical Technologies, Tlemcen Algeria 5-7 December 2019, ICHSMT'19.

\section{Authors' biography}

No Biography.

\section{References}

No references 